\title{
Therapy for fungal diseases: opportunities and priorities
}

\author{
David W. Denning and William W. Hope
}

School of Translational Medicine, Manchester Academic Health Science Centre, University of Manchester, University Hospital of South Manchester, Manchester M23 9LT, UK

\begin{abstract}
This article provides a perspective on the current status of drug therapy for invasive fungal diseases, together with priorities for the future development of novel compounds. Key opportunities for new drugs include production of orally bioavailable agents for the treatment of invasive aspergillosis, invasive candidiasis, cryptococcal meningitis and mucosal and urinary Candida infections. Orally bioavailable agents for the treatment of chronic pulmonary and allergic aspergillosis are also required, as well as new potent drugs against a range of medically important moulds. Antifungal resistance is a problem in certain contexts, but is generally less of a problem than bacterial infections. Earlier and more complete mycological diagnosis and improvements in underlying risk estimation will improve outcomes. The limitations of the current antifungal agents and opportunities for new developments are discussed.
\end{abstract}

\section{Fungal infections: the challenges ahead}

Invasive fungal infections are an increasing threat to human health. In the developed world, these infections predominantly occur in the context of increasingly aggressive immunosuppressive therapies. The overall mortality for invasive diseases caused by Candida spp. and Aspergillus spp. is $30-50 \%$, despite the advent of new diagnostic and therapeutic strategies. In the developing world, there are $\sim 1$ million cases of cryptococcal disease per year, resulting in 675000 deaths [1]. Allergic fungal syndromes are increasingly recognised [2]. Continued efforts are required to improve the often suboptimal therapeutic outcomes associated with fungal infections.

The high degree of phylogenetic relatedness between fungi and humans means that there are relatively few differential targets to be exploited for antifungal drug development. Fungi are involved in an interminable struggle for survival with each other and with other microbes. They produce a vast array of extracellular enzymes and secondary metabolites to counteract and digest the external world. Many antimicrobial agents have been isolated from fungi themselves [3]. The best example is penicillin, which was isolated from Penicillium notatum (now Penicillium chrysogenum) by Fleming, and later purified for medical use by Florey and Chain [4]. Similarly, the echinocandins, a novel class of antifungal compounds now in widespread clinical use, are semisynthetic derivatives of fungal-derived cyclic hexapeptides.

Corresponding author: Denning, D.W. (ddenning@manchester.ac.uk).
Currently, five classes of antifungal agents are used orally or intravenously for the treatment of fungal infections in humans: polyenes, pyrimidine analogues, allylamines, azoles and the echinocandins (Table 1). Each antifungal compound has advantages and limitations related to its spectrum of activity (Table 2), route of administration, drug interactions and toxicity profile. This review will describe the role and limitations of these agents for the treatment of the most common and medically important syndromes. Agents that are in early phases of development will not be discussed. We reflect on the challenges and opportunities for the development of novel therapeutic strategies for the most common fungal syndromes as a way of improving outcomes. Our key insights for drug development and future research are summarised in Box 1 and Box 2 .

\section{Antifungal compounds \\ Polyenes}

The polyenes are broad-spectrum antifungal agents produced by the bacterial genus Streptomyces. Nystatin was discovered in 1950 from the fermentation broth of Streptomyces noursei, and is still used as a topical antifungal agent. This was followed by isolation of fracidin, rimocidin, endomycin, ascosin, trichomycin and antimycoin in the early $1950 \mathrm{~s}$ [5]. Amphotericin A and B were isolated from $S$. nodosum and reported in 1955 [6], but only amphotericin B was developed because of its superior potency. Since then, $\sim 90$ polyenes have been discovered, but problems with solubility, stability, oral bioavailability and toxicity have prevented many of these compounds being developed for clinical purposes [5]. Natamycin is still widely used as a topical ophthalmic agent.

The complex chemistry and systemic toxicity of the polyenes provided the impetus to develop novel drug delivery systems that enable systemic therapy [7]. The best example of this is the lipid formulations of amphotericin B, although a liposomal formulation of nystatin was also developed [8]. The current commercially available lipid preparations of amphotericin B include amphotericin B lipid complex (ABLC), amphotericin $\mathrm{B}$ colloidal dispersion $(\mathrm{ABCD})$ and liposomal amphotericin B (Figure 1). Other formulations are in various stages of clinical development, including preparations that are orally bioavailable [9]. Lipid formulations differ significantly in terms of their pharmacokinetics, tissue distribution and toxicity profile [10,11]. Amphotericin B formulations are widely used for the treatment of disseminated candidiasis, invasive aspergillosis, cryptococcal meningitis, and infections caused by the Mucorales [12,13]. 
Table 1. Characteristics of antifungal agents used to treat invasive or allergic disease ${ }^{a}$

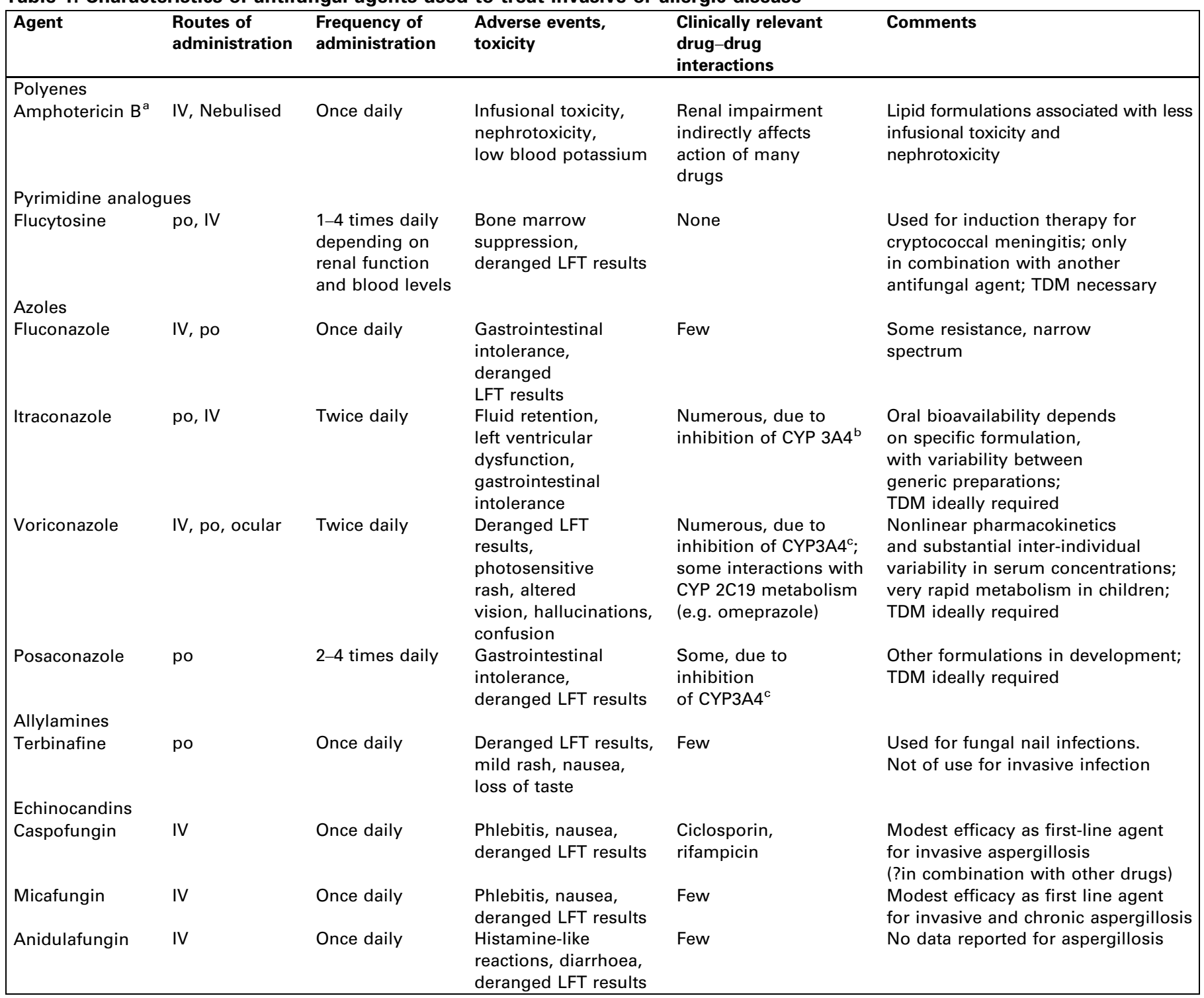

Abbreviations: CYP 3A4, Cytochrome P450 isozyme 3A4; IV, intravenous; LFT, liver function test; po, oral; TDM, therapeutic drug monitoring.

${ }^{a}$ Amphotericin B deoxycholate, liposomal amphotericin B, amphotericin B lipid complex or amphotericin B colloid dispersion.

${ }^{b}$ The compound inhibits CYP $3 A 4$ and leads to higher concentrations of drugs metabolised by this enzyme; low concentrations with compounds that accelerate metabolism through CYP $3 A 4$ (e.g. rifampicin, anticonvulsants).

\section{Pyrimidine analogues}

Flucytosine (5-fluorocytosine; 5FC) was discovered in 1964 by Roche Laboratories within an anti-neoplastic drug development programme. Flucytosine does not have any intrinsic anti-neoplastic activity, but is active against medically important yeasts such as Candida spp. and Cryptococcus spp., and against a limited number of moulds such as Aspergillus spp. and agents of chromoblastomycosis $[14,15]$.

Flucytosine is a pyrimidine analogue, which acts as a subversive substrate within the pyrimidine salvage pathway that is responsible for scavenging precursors for nucleic acid synthesis. Flucytosine is transported into fungal cells by cytosine permeases, where it is then deaminated to 5 -fluorouracil (5FU) by a fungal enzyme, cytosine deaminase. Sequential phosphorylation of $5 \mathrm{FU}$ produces toxic fluorinated nucleotides that ultimately interfere with DNA and RNA synthesis. [16]. The differential antifungal activity of flucytosine results from the absence of cytosine deaminase in humans (Figure 2).

In vitro studies described the emergence of resistance to flucytosine with concentrations $<25 \mathrm{mg} \mathrm{L}^{-1}$ [17], leading to the longstanding dogma that flucytosine should always be used in combination. Flucytosine causes bone marrow suppression, especially with peak plasma concentrations that are persistently $>100 \mathrm{mg} \mathrm{L}^{-1}$, which is the primary reason why therapeutic drug monitoring is essential [18]. Flucytosine (in combination with another antifungal agent) is the standard of care for the treatment of cryptococcal meningitis [19], but is also occasionally used (in combination) for the treatment of invasive candidiasis at sites where drug penetration might be suboptimal (e.g. the eye or central nervous system), or to treat infections that are resistant or refractory to first-line antifungal agents. 
Table 2. Relative in vitro and clinical activity of antifungal agents for more common and important invasive fungal pathogens ${ }^{a}$

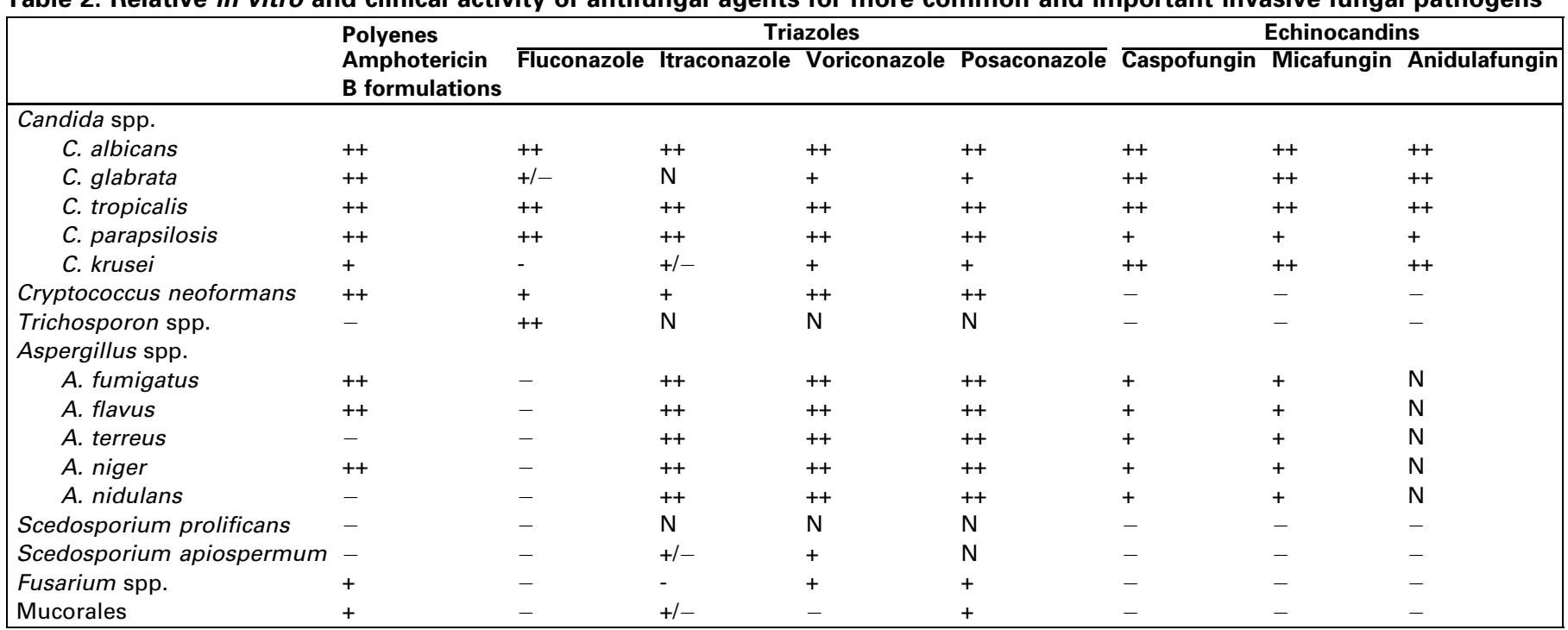

Relative activity is indicated as follows:,- no activity; + /-, slight activity; +, modest activity; ++, good activity; $\mathrm{N}=$ no data.

${ }^{a}$ Most fungi causing cutaneous and allergic disease have been excluded.

\section{The imidazoles and triazoles}

Benzimidazole, the first azole, was discovered in 1944 and demonstrated to be active against yeasts and several bacterial species. Approximately 100 derivatives of this compound were subsequently synthesised. Several decades later, drug discovery programmes at Bayer led to the discovery of the tritylimidazole derivative clotrimazole. Phenethyl imidazole derivatives developed by Janssen yielded miconazole, econazole and ketoconazole. Ketoconazole was the first orally bioavailable imidazole [20]. Despite hepatotoxicity, inhibition of endogenous human cortisol synthesis and a multitude of drug interactions, ketoconazole revolutionised the treatment of oral candidiasis, coccidioidomycosis, endemic mycoses, dermatophyte infections and veterinary fungal infections. Subsequently, many azoles for agricultural, veterinary and human use were developed. Modifications of the active ring produced the triazoles, and members of this class in

Box 1. Priorities for development of novel antifungal agents for the treatment of invasive fungal infections

- Oral compound with activity against all common Candida species (including triazole-resistant strains).

- Parenteral or oral compounds with activity against Cryptococcus neoformans and penetration into the central nervous system.

- Parenteral and oral compounds with activity against Aspergillus spp., including triazole-resistant species. Ideally, compounds should have few drug interactions, and should be safe in patients with renal or hepatic impairment.

- Parenteral and oral compounds with activity against rare, but medically important moulds (e.g. Mucorales, Scedosporium spp.).

- Oral agent(s) for the treatment of chronic pulmonary and allergic aspergillosis, with few drug interactions (especially with corticosteroids) and favourable intrapulmonary pharmacokinetics.

- Development of novel formulations of existing compounds that have a more favourable pharmacokinetic properties (e.g. enhanced oral bioavailability)

- Formulations that enable novel uses of existing compounds (e.g. aerosolisation) current clinical use include fluconazole, itraconazole, voriconazole and posaconazole; these are discussed below.

The putative antifungal target for the azoles is the enzyme 14- $\alpha$ demethylase. The common imidazole or triazole ring (Figure 2) interacts with the haem moiety of this enzyme; whereas, the side chain orientates the active site of the molecule to the haem moiety within the target protein. The interaction of the side chain within the binding pocket is responsible for the differential antifungal activity that is characteristic of these compounds. Inhibition of 14- $\alpha$ demethylase disrupts the synthesis of sterols that are required for normal fungal membrane function.

Fluconazole Fluconazole exhibits linear pharmacokinetics (i.e. the dose is directly proportional to the area under the concentration-time curve) [21], is water-soluble, and is very well tolerated. Despite its frequently reduced or absent activity against Candida glabrata and the inherent resistance of Candida krusei, along with the absence of activity against Aspergillus spp. and other medically important moulds, fluconazole is extensively used for the prevention and treatment of superficial and invasive Candida infections [22,23]. Fluconazole is also used for the treatment of cryptococcal infections and has demonstrated efficacy against dermatophytes [24].

Itraconazole Itraconazole was the first orally bioavailable agent with anti-Aspergillus activity [25]. Human metabolism of itraconazole produces a metabolite, hydroxyitraconazole, which has comparable antifungal activity to the parent. Itraconazole is available as capsules for oral use, and as a cyclodextrin formulation for oral and intravenous use. The major limitation of the capsule formulation is its highly variable acid-dependant oral bioavailability [26]. This often leads to subtherapeutic concentrations in critically ill patients with hypochlorohydria (elevated gastric $\mathrm{pH}$ ). Itraconazole 
Box 2. Priorities for future research fungal to improve the outcomes of invasive fungal infections

- Improved diagnostic tests, with high accuracy, rapid turnaround time, and prognostic value that can guide antifungal therapy in real time.

- Direct detection of species causing infection and antifungal resistance, without having to first culture the fungus.

- Better risk prediction models, including genetic risk factors to target surveillance, prophylaxis and rapid diagnostics more appropriately.

- Mechanisms to ensure the attainment of maximal antifungal effect as quickly as possible (e.g. combination therapy, therapeutic drug monitoring).

- Novel immunomodulatory treatments to maximise antifungal effect and minimise immune-mediated damage and fibrosis.

- Collaborative national and international programmes for antifungal resistance surveillance.

solution results in improved oral bioavailability, but is often associated with gastrointestinal intolerance. The intravenous formulation circumvents problems with variable oral bioavailability, but is not widely used. The administration of itraconazole solution (not capsules) and serum trough concentrations $>0.5 \mathrm{mg} \mathrm{L}^{-1}$ (measured by HPLC) decreases the incidence of invasive fungal infections in patients with profound and prolonged neutropenia [27]. Itraconazole is used for the treatment of chronic pulmonary aspergillosis, allergic syndromes related to Aspergillus spp., onychomycosis, histoplasmosis, blastomycosis and sporotrichosis [28]. Itraconazole has activity against Candida albicans, and can be used for the treatment of both vulvovaginal or oral candidiasis caused by fluconazole-resistant strains, providing there is no laboratory evidence of cross-resistance. Itraconazole has no dependable activity against the Mucorales, although on occasions successful treatment has been documented [29]. The use of itraconazole is associated with a number of toxicities, which increase with increasing drug exposure [30].

Voriconazole, a structural congener of fluconazole, is active against Aspergillus spp., Fusarium spp. and Candida spp. (including the fluconazole-resistant or less susceptible species of $C$. glabrata and C. krusei). Voriconazole has no activity against the Mucorales, but is a firstline agent for the treatment of invasive aspergillosis, and is superior to amphotericin B deoxycholate for initial therapy of this syndrome [31]. Voriconazole is not currently licensed for the prophylaxis of fungal infection, or for treatment of persistently febrile patients with low neutrophil numbers who have suspected, but unproven infection [32]. Its clinical use is complicated by a number of drug interactions, highly variable pharmacokinetics and more side effects than those of fluconazole.

Posaconazole is structurally related to itraconazole, and has broad spectrum activity against Candida spp., Aspergillus spp. and the Mucorales [33]. Oral bioavailability is increased by food, especially by fatty food [34,35]. Capsule and intravenous preparations are in development. There are fewer drug interactions compared with itraconazole or voriconazole. Posaconazole is effective for preventing fungal infections for neutropenic patients with leukaemia and for recipients of bone marrow transplants who have graftversus-host disease [36,37]. This compound is also licensed for second-line therapy for patients with invasive aspergillosis [38], and is partially effective for the treatment of infections caused by the Mucorales [39]. Posaconazole is usually well tolerated.

\section{Allylamines}

Terbinafine, the only allylamine in clinical use, was discovered in a medicinal chemistry programme as a derivative of the topical antifungal naftifine, which was the original compound of the allylamine class. The target for terbinafine, squalene epoxidase, was identified in 1984 [40]. Terbinafine is the agent of choice for many dermatophyte infections including onychomycosis [41]. Although it has limited activity for treatment of invasive fungal diseases, terbinafine can be combined with voriconazole for the treatment of infections caused by Scedosporium prolificans [42].

\section{Echinocandins}

The echinocandins are semi-synthetic cyclic lipohexapeptides with molecular weights of $\sim 1200 \mathrm{Da}$; which were originally derived from fungi [43]. Cilofungin was the first echinocandin used in clinical trials, but its development was abandoned because of toxicity. Caspofungin, micafungin and anidulafungin are active against Candida spp. and Aspergillus spp., but few other fungi. The echinocandins have a unique mechanism of action via the non-competitive inhibition of the cell wall protein 1,3- $\beta$-D-glucan synthase (Figure 2). Decreased 1,3- $\beta$-D-glucan concentrations in the cell wall result in osmotic fragility and lysis of Candida spp. Although the echinocandins do not kill Aspergillus spp., they induce morphological changes within these fungi and decrease their propensity to cause pulmonary injury [44]. The currently licensed echinocandins are not orally bioavailable.

\section{Therapy for Candida syndromes}

Candida spp. are human commensals that can cause superficial and invasive infection. Despite improved understanding of the pathogenesis of invasive disease and the advent of new diagnostic and therapeutic strategies, the attributable mortality has essentially remained unchanged for the past few decades at $\sim 40 \%[45,46]$.

\section{Disseminated candidiasis}

Disseminated candidiasis (candidaemia and invasive candidiasis) is a life-threatening syndrome with an attributable mortality of $10-50 \%$ [46]. Although amphotericin B deoxycholate is an effective agent for this syndrome, its use is limited by both infusional toxicity and nephrotoxicity. Liposomal amphotericin B is one of the first-line agents for the treatment of disseminated candidiasis [13].

Fluconazole has been extensively used for the treatment of this syndrome, but since the introduction of the echinocandins, its role as a first-line agent is less clear. A recent clinical trial comparing anidulafungin with fluconazole suggested that anidulafungin results in superior outcomes at the end of therapy [47]. First-line use of fluconazole is reasonable for patients who (i) are haemodynamically 


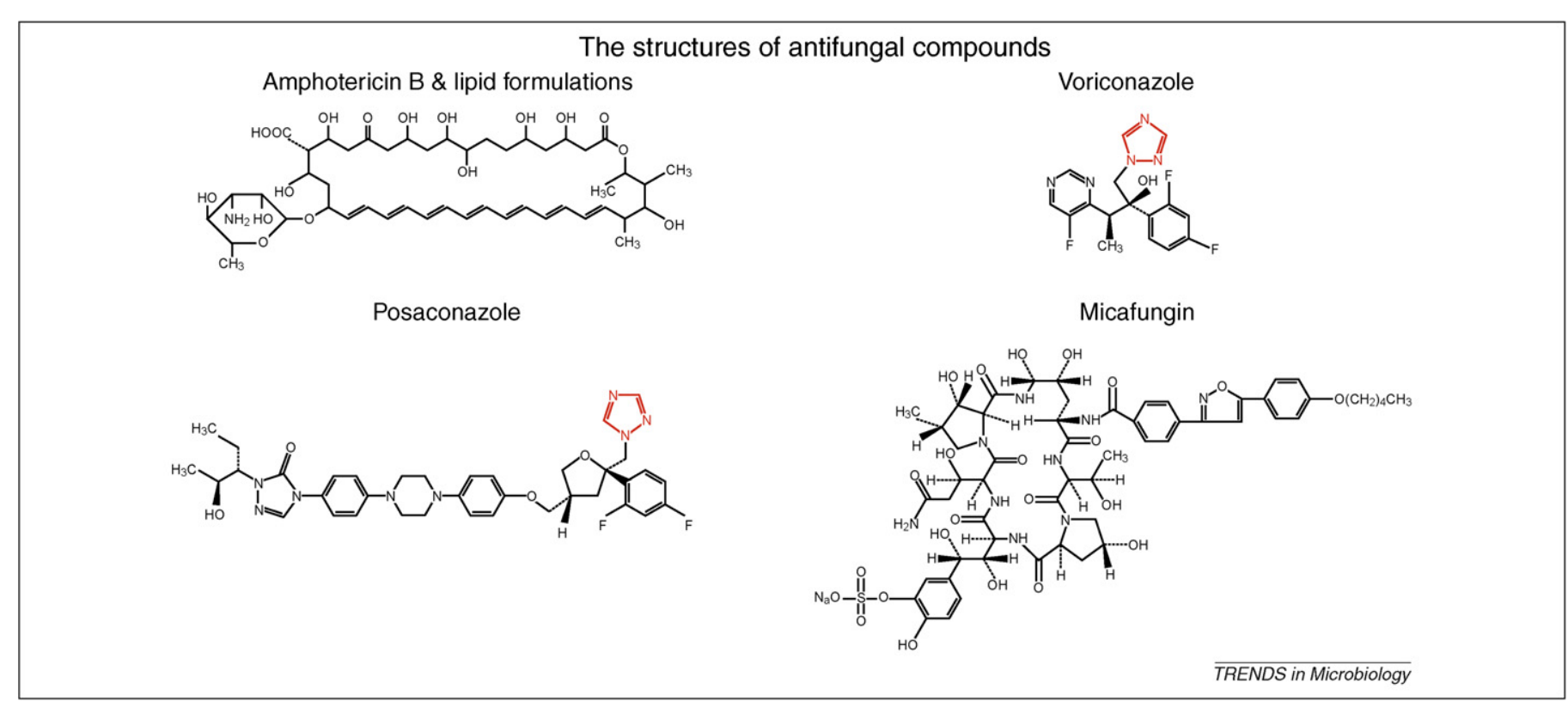

Figure 1. The structures of selected antifungal agents within each of the major antifungal classes.

stable; (ii) have not been previously exposed to triazoles; and (iii) have a low estimated risk of infection with $C$. glabrata and $C$. krusei based on local epidemiological patterns. Fluconazole can be used after initial parenteral therapy in many patients to complete 2 weeks of antifungal therapy after negative blood cultures. Voriconazole is an effective agent for disseminated candidiasis [48], but its use is often limited by drug interactions and (in the case of the intravenous formulation) accumulation of the cyclodextrin excipient in patients with a glomerular filtration

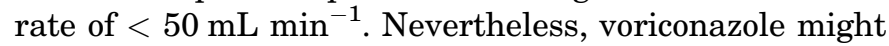
have activity against fluconazole-resistant $C$. glabrata and C. krusei, and is a potential agent for oral therapy in this circumstance.

The echinocandins are increasingly used for the treatment of disseminated candidiasis. They are as effective as amphotericin B [13,49] and probably superior to fluconazole [47]. They are effective against organisms with intrinsic or acquired triazole resistance, can be safely used in patients with renal and hepatic insufficiency, and have few drug interactions. The minimum inhibitory concentrations of C. parapsilosis, C. guilliermondii and C. famata are higher than those of other species, but the clinical relevance of this phenomenon is unclear [50,51]. Echinocandin resistance resulting from amino acid substitutions within defined regions of the target protein Fks1 is increasingly recognised, but is usually only observed following prolonged therapy [52,53]

Challenges and opportunities for the treatment of disseminated candidiasis are (i) improved diagnostics (earlier therapy is associated with improved mortality) [54]; (ii) potential advantage of immunisation [55]; and (iii) better risk stratification, especially for failure of fluconazole prophylaxis.

\section{Candidiasis of the renal tract}

The presence of Candida in the urine (candiduria) occurs in $\sim 9 \%$ of hospitalised patients $[56,57]$, and might be the result of simple colonisation of the lower renal tract, of upper renal tract disease or of 'spill' from bloodstream infection [58]. The majority of patients with asymptomatic candiduria do not require antifungal therapy. Removal (or replacement) of an indwelling urinary catheter, if present, is usually effective [59]. If therapy is deemed necessary, fluconazole is the agent of choice [60,61]. Bladder washouts with amphotericin B are an alternative for fluconazole-resistant infections. Flucytosine achieves high urinary concentrations, but relapse is common. If candiduria is a manifestation of (suspected or proven) candidaemia, systemic therapy is warranted, even if the chosen compound does not achieve high urinary concentrations. Safe and well-tolerated agents with activity against Candida in the urinary tract would be useful for the modest number of patients for whom specific therapy is indicated.

\section{Oral and oesophageal candidiasis}

Oral and oesophageal candidiasis predominantly occurs in patients with AIDS, but occasionally in other settings. While oral candidiasis can often be managed with topical therapy (e.g. clotrimazole, miconazole, nystatin, amphotericin B lozenges), oesophageal candidiasis requires systemic antifungal therapy. Fluconazole is safe and effective, and is the initial systemic treatment of choice. A single dose of $400 \mathrm{mg}$ is as effective as giving $150 \mathrm{mg}$ for 14 days [62].

If there is resistance to fluconazole, the use of an alternative triazole is possible, but a polyene or an echinocandin is required if triazole cross-resistance is present [63-65]. Although echinocandins are effective agents for the treatment of oesophageal candidiasis, the proportion of patients that relapse tends to be higher compared with the triazoles. Echinocandin resistance can develop after prolonged therapy, with mutations within Fks1 being responsible. An orally bioavailable agent with activity against triazole-resistant Candida spp. would be useful. 


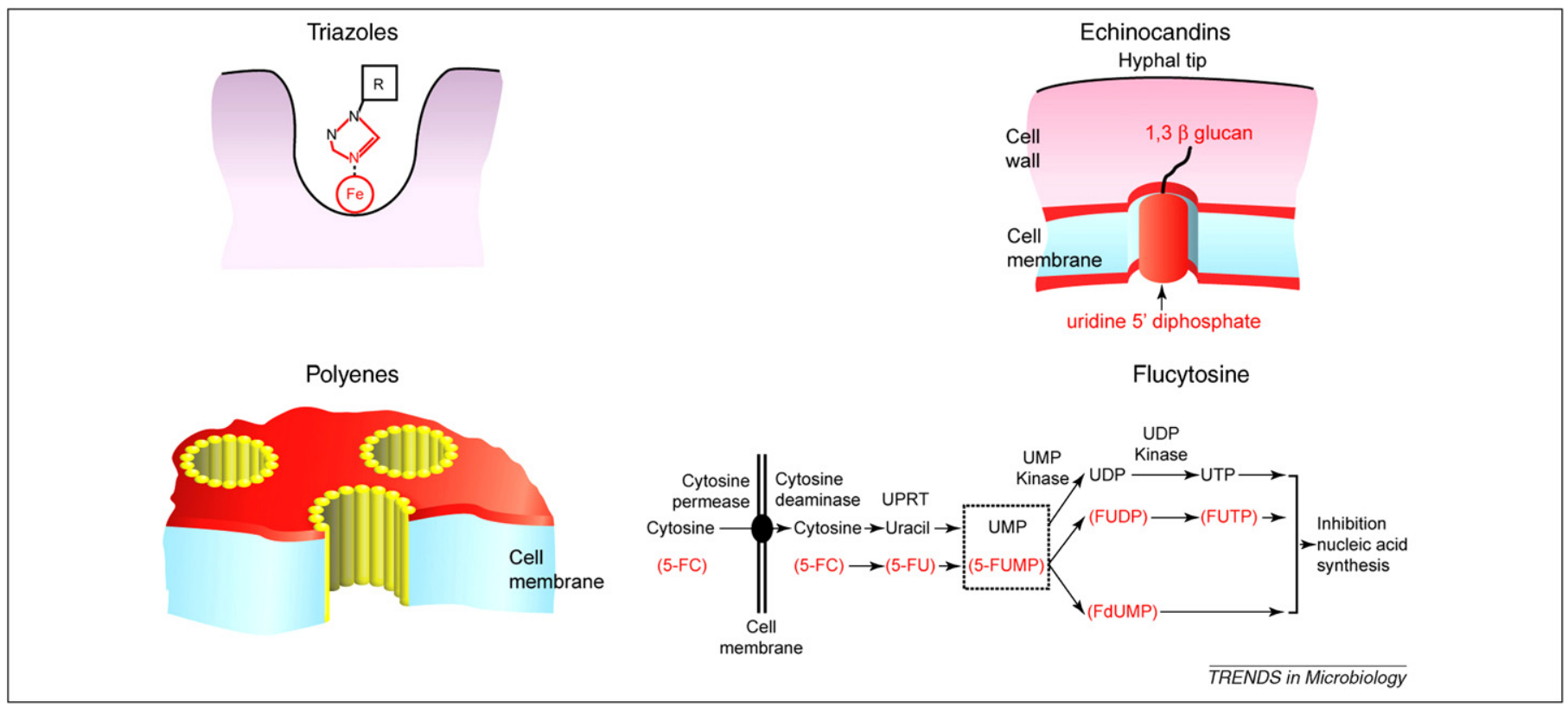

Figure 2. Modes of action of the main classes of antifungal agents used to treat invasive fungal infections. (a) The active site of the triazoles contacts a haem moiety at the

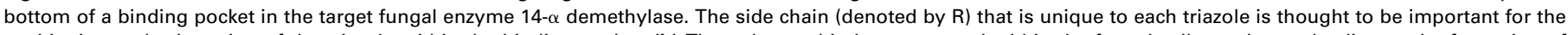

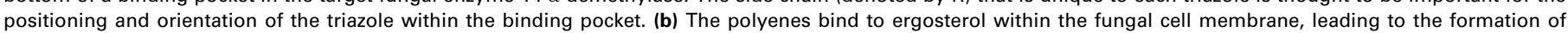

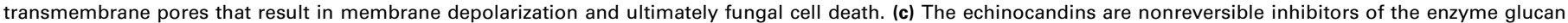

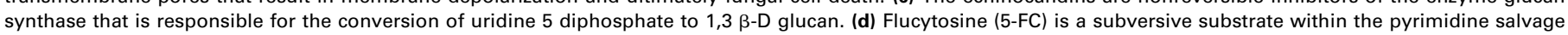
pathway. The normal pathway and the toxic fluorinated metabolites are shown. Fluorinated nucleotides lead to disruption of nucleic acid synthesis.

\section{Vulvovaginal candidiasis}

Vulvovaginal candidiasis is common in pre-menopausal women. Most patients respond promptly to antifungal therapy, but some have recurrent infection or persistent symptoms. Women with cystic fibrosis are especially prone to recurrent infection, probably because systemic antibacterial therapy disrupts the normal vaginal flora [66]. Nystatin can be effective for the treatment of fluconazole-resistant organisms (usually caused by $C$. glabrata), but local application of a weak solution of boric acid or amphotericin B and flucytosine pessaries are alternatives $[67,68]$. The triazoles are contraindicated in pregnancy [69] and therefore, prolonged courses of triazoles can interfere with plans for conception in women of childbearing age with recurrent vulvovaginal candidiasis. During pregnancy, topical therapy is the only option. A systemic antifungal therapy for vulvovaginal candidiasis caused by C. glabrata and that is safe in pregnancy would be particularly useful.

\section{Treatment of infections caused by Aspergillus spp}

Aspergillus fumigatus is a leading cause of life-threatening mould infection in immunocompromised patients. Humans are continuously exposed to this environmentally ubiquitous mould. A. fumigatus is unique for the extent of clinical syndromes it produces, which range from allergic syndromes through chronic invasive forms and acute rapidly progressive life-threatening infection. Other species of Aspergillus cause some similar syndromes. Aspergillus terreus and Aspergillus nidulans are resistant to amphotericin B. Triazole resistance has been documented in environmental strains of A. fumigatus [70] and during treatment [71].

\section{Invasive aspergillosis}

Invasive aspergillosis is associated with $\sim 50 \%$ mortality, despite a better understanding of pathogenesis and the advent of new diagnostic and therapeutic methods. This syndrome remains extremely difficult to treat, and is often diagnosed relatively late. The majority of patients are treated presumptively, without definitive microbiological evidence.

One of the significant therapeutic advances in the past decade is an improved understanding of dose-response relationships for the polyenes. Early studies suggested that improved responses were possible using high dosages of amphotericin B deoxycholate (e.g. 1-1.5 mg kg${ }^{-1}$ ) [72]. Despite few data, this approach was widely adopted. Unfortunately, however, these dosages are accompanied by significant toxicity, which is independently associated with higher mortality, requirement for haemodialysis and excessive hospital costs [73,74].

The lipid formulations of amphotericin B are less toxic than deoxycholate. Despite the ability to be able to administer more active drug, there is no direct evidence that these preparations are more effective than amphotericin B deoxycholate. Several clinical trials have enabled effective dosages of liposomal amphotericin B to be identified $[11,12,75]$. Although the optimal dosage of liposomal amphotericin B is not known, most authors advocate $3 \mathrm{mg} \mathrm{kg}{ }^{-1}$ for invasive aspergillosis.

Another major advance has been the introduction of triazoles with anti-Aspergillus activity. The efficacy and safety of itraconazole for invasive aspergillosis was established in an early noncomparative trial with approximately $33 \%$ of patients responding to therapy [76] Voriconazole [followed by other licensed antifungal 
therapy (OLAT)] is superior to amphotericin B deoxycholate (followed by OLAT) in terms of efficacy, improved survival and the incidence of adverse events (53\% versus $32 \%$ response rate, respectively) [77]. Other studies support favourable outcomes of voriconazole therapy for patients with invasive aspergillosis [78,79]. Posaconazole is effective for the treatment of patients who are refractory to or intolerant of other antifungal agents, but its use as a first line agent has not been established [38].

The echinocandins have both in vitro and in vivo activity against Aspergillus spp. [43]. Hyphae exposed to echinocandins are gnarled, swollen and excessively branched, but remain viable. The echinocandins produce a modest survival advantage in laboratory animal models of invasive pulmonary aspergillosis, despite the fungal burden in the lung being unchanged or paradoxically higher. In echinocandin-treated laboratory animals, the fungi appear to have a reduced propensity for causing pulmonary injury [44]. Increasing evidence suggests that the echinocandins cause unmasking of immunoreactive fungal epitopes that might facilitate cooperative immunological killing [80-82]. Unfortunately, the optimal echinocandin dosage for invasive aspergillosis is not known and is very difficult to determine using current preclinical data. The echinocandins are potentially useful for salvage therapy, but their role in the primary treatment of invasive aspergillosis is less clear.

There are several challenges in the treatment of invasive aspergillosis: (i) many diagnoses are established relatively late, often when there has been significant tissue damage; (ii) the biomarkers with prognostic value that can be used to follow antifungal therapy in real time are only rudimentary; (iii) there is often uncertainty about the diagnosis because of the suboptimal specificity of current diagnostic methods; in many cases a syndrome that is consistent with an invasive fungal infection is assumed to be invasive aspergillosis without any microbiological data available to enable a diagnosis to be established at either a genus or species level; (iv) emerging triazole resistance. Therefore, the opportunities are as follows: (i) development of improved diagnostics; (ii) development of drugs that might have an effect upon pathogenesis (e.g. compounds that inhibit angioinvasion or prevent dissemination); (iii) further study of the role of adjuvant treatments (e.g. surgery, granulocyte transfusions and immunotherapy); (iv) development of strategies that enable the attainment of near maximal antifungal effect as quickly as possible (e.g. innovative dosing strategies, therapeutic drug monitoring and combination antifungal therapy); (iv) development of safe, orally bioavailable compounds with broad spectrum anti-Aspergillus activity, which are devoid of drug interactions and have a low propensity for development of resistance; and (v) development of prognostic biomarkers that enable real-time monitoring of antifungal therapy.

\section{Chronic pulmonary aspergillosis and aspergilloma}

Chronic pulmonary aspergillosis is a slowly progressive infection that is characterised by prominent constitutional and respiratory symptoms, progressive development of cavities in the lung, and the presence of anti-Aspergillus IgG and IgE antibodies [83-85]. This syndrome invariably occurs in the context of pre-existing structural lung disease, and many patients have innate immunological defects [8688]. An aspergilloma (fungal ball) within pulmonary cavities can be intermittently present $[84,85]$.

Most patients with chronic pulmonary aspergillosis require long-term maintenance antifungal therapy (i.e. months to years). Despite some limitations, the triazoles are the only oral agents available for this purpose [89]. Of considerable concern is the recent emergence of triazole resistance $[71,90]$. Isolated resistance to itraconazole is commonly observed, but cross-resistance to voriconazole and/or posaconazole occurs in $>50 \%$ of instances. Infections by strains resistant to all triazoles require parenteral therapy with amphotericin B or an echinocandin.

The challenges to be overcome include: (i) the lack of knowledge of the optimal triazole regimen; and (ii) the fact that regimens developed for acute invasive disease might not achieve high concentrations within pulmonary cavities and therefore might promote antifungal resistance. Opportunities for future therapy include: (i) the need to identify regimens that do not promote the generation of antifungal resistance; (ii) the development of orally bioavailable compounds without cross-resistance to the triazoles, which are suitable for long-term use; (iii) further definition of the role of adjunctive immunotherapy; (iv) rapid, direct methods to identify triazole resistance, as cultures are often negative; and (v) strategies to minimise pulmonary fibrosis.

\section{Allergic bronchopulmonary aspergillosis and severe asthma with fungal sensitisation}

Patients with either allergic bronchopulmonary aspergillosis (ABPA) or severe asthma with fungal sensitisation (SAFS) appear to benefit from itraconazole treatment [91-93]. However, approximately $15 \%$ of patients cannot tolerate itraconazole for months [93]. Relapses are common, and an interaction between itraconazole and inhaled corticosteroid often leads to higher reduced metabolism of corticosteroid, thus increasing corticosteroid side-effects. Because long-term therapy is required for both ABPA and SAFS, safe and well-tolerated drugs are essential. Although A. fumigatus is the most common fungus involved, others such as Alternaria alternata and Cladosporium herbarum are implicated, requiring a slightly different antifungal spectrum compared with agents required for invasive disease. Broad-spectrum, orally bioavailable, mould-active agents would potentially enable more effective treatment of patients with these allergic syndromes.

\section{Cryptococcal meningitis}

Cryptococcal meningitis occurs in a wide range of immunocompromised patients with defects of cellular immunity. The global burden of infection largely resides with patients with AIDS living in resource-poor settings. Patients with high infectious burden and raised intracranial pressure tend to have a worse prognosis. Induction therapy with amphotericin B deoxycholate $0.7-1.0 \mathrm{mg} \mathrm{kg}^{-1}$ combined with flucytosine $100 \mathrm{mg} \mathrm{kg}^{-1}$ per day for 2 weeks, followed by further consolidation therapy with fluconazole is considered standard care in the developed world [19]. Management of raised intracranial pressure is important.

Recent studies suggest that the fungal burden in the cerebrospinal fluid has prognostic significance, and can be 
used as biomarker for antifungal therapy. This has facilitated several clinical trials examining innovative regimens. The inability to administer amphotericin $B$ deoxycholate in some resource-poor settings has led to attempts to optimise oral induction regimens. Fluconazole $1200 \mathrm{mg}$ per day is more rapidly fungicidal than $800 \mathrm{mg}$, but is still associated with $54 \%$ mortality at 10 weeks [94]. The addition of flucytosine to fluconazole $1200 \mathrm{mg}$ per day results in greater fungicidal activity compared with fluconazole monotherapy [95]. The effect of this combination in comparison with amphotericin B is not known, but further studies are planned.

The challenges and opportunities for the treatment of cryptococcal meningitis are: (i) dramatically increased levels of funding for this global health problem; (ii) further refinement of optimal regimens for induction and maintenance therapy, including oral options and other innovative regimens; (iii) development of new orally bioavailable drugs that penetrate the central nervous system; (iv) increasing the availability of oral flucytosine and other compounds in resource-poor settings; and (v) development of oral bioavailable formulations of amphotericin B.

\section{Mucorales and other rare moulds}

The Mucorales cause life-threatening infection of the sinuses, lungs, brain, gut, skin and kidney. Only amphotericin $\mathrm{B}$ formulations and posaconazole are reliably active against these organisms. When combined with aggressive surgery, the response rates to these agents is approximately $70 \%[39,96]$. An understanding of optimal antifungal therapy for these infections has been hampered by difficulties in predicting the in vivo responses to polyenes or posaconazole based on in vitro susceptibility tests and in developing suitable laboratory animal models that are faithful mimics of human disease. The widespread use of voriconazole, which has no inherent activity, is associated with an increase in the incidence of infection in some studies [97]. However, the combination of a polyene and echinocandin might be effective [98]. Iron chelation therapy is another possible therapeutic option [99].

There are increasing reports of other moulds that cause devastating infections in a range of patients, including dematiaceous (e.g. Curvularia and Bipolaris) [100] and hyaline moulds (e.g. Fusarium). These infections are likely to remain problematic unless new active agents active against common yeasts and moulds incidentally have an extended spectrum of activity.

Challenges and opportunities include: (i) development of international networks that enable collective experience of these infections and facilitation of clinical trials; (ii) the development of laboratory animal models that enable in vivo-to-human bridging studies that can be used to identify suitable regimens in the absence of large randomised clinical trial data; (iii) development of adjunctive treatments (e.g. iron chelation); and (iv) further development of novel antifungal combinations.

\section{Concluding remarks and future directions}

Although there have been major advancements in antifungal therapy, there is an ongoing requirement for new antifungal agents. Orally bioavailable and well-tolerated alternatives to the triazoles are urgently required. Several possible antifungal product profiles are shown in Box 1 (not in priority order). Not all novel antifungal agents necessarily need to have a broad spectrum of activity, but excellent activity against either Candida or Aspergillus is required for a significant economic return. Antifungal therapy of fungal allergic syndromes, including eosinophilic fungal rhinosinusitis, requires further study.

Several other requirements for further research are shown in Box 2. Accurate diagnostic tests with rapid turn around time will facilitate clinical trials. Triazole and echinocandin resistance is of concern, and requires more rapid approaches to detection and continued surveillance. Understanding which patients are at highest risk of developing a life-threatening infection is at present a major deficiency, and genetic markers will probably assist in risk assessment. Both combination antifungal therapy and immunomodulatory approaches require detailed study to maximise survival, and minimise tissue damage and fibrosis. Despite major immunosuppression, vaccines to prevent infection require study, and might be useful for improving therapeutic outcomes.

\section{Conflict of interest statement}

In the past 5 years, DD has had received grant support from Astellas, Merck, Pfizer, F2G, OrthoBiotech, Indevus, Basilea, the Fungal Research Trust, the Wellcome Trust, the Moulton Trust, The Medical Research Council, The Chronic Granulomatous Disease Research Trust, the National Institute of Allergy and Infectious Diseases and the European Union. He has been an advisor/consultant to Basilea, Vicuron (now Pfizer), Pfizer, Schering Plough (now Merck), Indevus, F2G, Nektar, Daiichi, Sigma Tau, Astellas, Gilead and York Pharma. He has been paid for talks on behalf of Schering, Astellas, Merck, Dainippon, AstraZeneca, Myconostica and Pfizer. He holds founder shares in F2G Ltd and Myconostica Ltd, both University of Manchester spin-out companies. WH has been an advisor or consultant to Astellas, Pfizer, Gilead, Schering and Vectura. He has received research support from Basilea, Astellas, Gilead and, Schering, and has been paid for talks on behalf of Pfizer, Gilead, Astellas and Merck.

\section{Acknowledgements}

WH is funded by a National Institute of Health Research (NIHR) Clinician Scientist Fellowship. We are indebted to Elaine Bradley of UHSM who drew the figures.

\section{References}

1 Park, B.J. et al. (2009) Estimation of the current global burden of cryptococcal meningitis among persons living with HIV/AIDS. Aids $23,525-530$

2 Agarwal, R. et al. (2009) Aspergillus hypersensitivity and allergic bronchopulmonary aspergillosis in patients with bronchial asthma: systematic review and meta-analysis. Int. J. Tuberc. Lung Dis. 13, 936-944

3 Vicente, M.F. et al. (2003) Microbial natural products as a source of antifungals. Clin. Microbiol. Infect. 9, 15-32

4 Chain, E. et al. (1940) Penicillin as a chemotherapeutic agent. Lancet 236, 226-228

5 Medoff, G. and Kobayashi, G.A. (1980) The Polyenes in Antifungal Chemotherapy. John Wiley \& Sons 
6 Donovick, R. et al. (1955) Amphotericins A and B, antifungal antibiotics produced by a streptomycete. I. In vitro studies. Antibiot. Annu. 3, 579-586

7 Torrado, J.J. et al. (2008) Amphotericin B formulations and drug targeting. J. Pharm. Sci. 97, 2405-2425

8 Groll, A.H. et al. (2003) Comparative drug disposition, urinary pharmacokinetics and renal effects of multilamellar liposomal nystatin and amphotericin B deoxycholate in rabbits. Antimicrob. Agents Chemother. 47, 3917-3925

9 Gershkovich, P. et al. (2009) Pharmacokinetics and biodistribution of amphotericin B in rats following oral administration in a novel lipidbased formulation. J. Antimicrob. Chemother. 64, 101-108

10 Groll, A.H. et al. (2006) Compartmentalized intrapulmonary pharmacokinetics of amphotericin B and its lipid formulations. Antimicrob. Agents Chemother. 50, 3418-3423

11 Wingard, J.R. et al. (2000) A randomized, double-blind comparative trial evaluating the safety of liposomal amphotericin B versus amphotericin B lipid complex in the empirical treatment of febrile neutropenia. L Amph/ABLC Collaborative Study Group. Clin. Infect. Dis. $31,1155-1163$

12 Cornely, O.A. et al. (2007) Liposomal amphotericin B as initial therapy for invasive mold infection: a randomized trial comparing a high-loading dose regimen with standard dosing (AmBiLoad trial). Clin. Infect. Dis. 44, 1289-1297

13 Kuse, E.R. et al. (2007) Micafungin versus liposomal amphotericin B for candidaemia and invasive candidosis: a phase III randomised double-blind trial. Lancet 369, 1519-1527

14 Verweij, P.E. et al. (2008) In vitro activities at pH 5.0 and pH 7.0 and in vivo efficacy of flucytosine against Aspergillus fumigatus. Antimicrob. Agents Chemother. 52, 4483-4485

15 Vandevelde, A.G. et al. (1972) 5-fluorocytosine in the treatment of mycotic infections. Ann. Intern. Med. 77, 43-51

16 Hope, W.W. (2010) Flucytosine (5-fluorocytosine; 5FC). In Kucers' the Use of Antibiotics

17 Normark, S. and Schonebeck, J. (1972) In vitro studies of 5fluorocytosine resistance in Candida albicans and Torulopsis glabrata. Antimicrob. Agents Chemother. 2, 114-121

18 Stamm, A.M. et al. (1987) Toxicity of amphotericin B plus flucytosine in 194 patients with cryptococcal meningitis. Am. J. Med. 83, 236-242

19 Perfect, J.R. et al. Clinical practice guidelines for the management of cryptococcal disease: 2010 update by the Infectious Diseases Society of America. Clin. Infect. Dis. 50, 291-322

20 Fromtling, R.A. (1988) Overview of medically important antifungal azole derivatives. Clin. Microbiol. Rev. 1, 187-217

21 McLachlan, A.J. and Tett, S.E. (1996) Pharmacokinetics of fluconazole in people with HIV infection: a population analysis. $\mathrm{Br}$. J. Clin. Pharmacol. 41, 291-298

22 Rex, J.H. et al. (1994) A randomized trial comparing fluconazole with amphotericin B for the treatment of candidemia in patients without neutropenia. Candidemia Study Group and the National Institute. $N$. Engl. J. Med. 331, 1325-1330

23 Slavin, M.A. et al. (1995) Efficacy and safety of fluconazole prophylaxis for fungal infections after marrow transplantation-a prospective, randomized, double-blind study. J. Infect. Dis. 171, 1545-1552

24 Dastghaib, L. et al. (2005) Therapeutic options for the treatment of tinea capitis: griseofulvin versus fluconazole. J. Dermatolog. Treat. 16, 43-46

25 Denning, D.W. et al. (1989) Treatment of invasive aspergillosis with itraconazole. Am. J. Med. 86, 791-800

26 Barone, J.A. et al. (1998) Food interaction and steady-state pharmacokinetics of itraconazole oral solution in healthy volunteers. Pharmacotherapy 18, 295-301

27 Glasmacher, A. et al. (2003) Itraconazole prevents invasive fungal infections in neutropenic patients treated for hematologic malignancies: evidence from a meta-analysis of 3,597 patients. J. Clin. Oncol. 21, 4615-4626

28 Zonios, D.I. and Bennett, J.E. (2008) Update on azole antifungals. Semin. Respir. Crit. Care. Med. 29, 198-210

29 Eisen, D.P. and Robson, J. (2004) Complete resolution of pulmonary Rhizopus oryzae infection with itraconazole treatment: more evidence of the utility of azoles for zygomycosis. Mycoses 47, 159-162

30 Lestner, J.M. et al. (2009) Toxicodynamics of itraconazole: implications for therapeutic drug monitoring. Clin. Infect. Dis. 49, 928-930
31 Walsh, T.J. et al. (2008) Treatment of aspergillosis: clinical practice guidelines of the Infectious Diseases Society of America. Clin. Infect. Dis. 46, 327-360

32 Walsh, T.J. et al. (2002) Voriconazole compared with liposomal amphotericin B for empirical antifungal therapy in patients with neutropenia and persistent fever. N. Engl. J. Med. 346, 225-234

33 Torres, H.A. et al. (2005) Posaconazole: a broad-spectrum triazole antifungal. Lancet Infect. Dis. 5, 775-785

34 Courtney, R. et al. (2004) Effect of food on the relative bioavailability of two oral formulations of posaconazole in healthy adults. Br. J. Clin. Pharmacol. 57, 218-222

35 Courtney, R. et al. (2004) Pharmacokinetics of posaconazole coadministered with antacid in fasting or nonfasting healthy men. Antimicrob. Agents Chemother. 48, 804-808

36 Cornely, O.A. et al. (2007) Posaconazole vs. fluconazole or itraconazole prophylaxis in patients with neutropenia. N. Engl.J. Med. 356, 348-359

37 Ullmann, A.J.et al. (2007) Posaconazole or fluconazole for prophylaxis in severe graft-versus-host disease. N. Engl. J. Med. 356, 335-347

38 Walsh, T.J. et al. (2007) Treatment of invasive aspergillosis with posaconazole in patients who are refractory to or intolerant of conventional therapy: an externally controlled trial. Clin. Infect. Dis. $44,2-12$

39 Greenberg, R.N. et al. (2006) Posaconazole as salvage therapy for zygomycosis. Antimicrob. Agents Chemother. 50, 126-133

40 Petranyi, G. et al. (1984) Allylamine derivatives: new class of synthetic antifungal agents inhibiting fungal squalene epoxidase. Science 224, 1239-1241

41 Sigurgeirsson, B. et al. (2002) Long-term effectiveness of treatment with terbinafine vs itraconazole in onychomycosis: a 5-year blinded prospective follow-up study. Arch. Dermatol. 138, 353-357

42 Gosbell, I.B. et al. (2003) Cure of orthopaedic infection with Scedosporium prolificans, using voriconazole plus terbinafine, without the need for radical surgery. Mycoses 46, 233-236

43 Denning, D.W. (2003) Echinocandin antifungal drugs. Lancet 362, 1142-1151

44 Petraitiene, R. et al. (2002) Antifungal efficacy of caspofungin (MK0991) in experimental pulmonary aspergillosis in persistently neutropenic rabbits: pharmacokinetics, drug disposition, and relationship to galactomannan antigenemia. Antimicrob. Agents Chemother. 46, 12-23

45 Gudlaugsson, O. et al. (2003) Attributable mortality of nosocomial candidemia, revisited. Clin. Infect. Dis. 37, 1172-1177

46 Hassan, I. et al. (2009) Excess mortality, length of stay and cost attributable to candidaemia. J. Infect. 59, 360-365

47 Reboli, A.C. et al. (2007) Anidulafungin versus fluconazole for invasive candidiasis. N. Engl. J. Med. 356, 2472-2482

48 Kullberg, B.J. et al. (2005) Voriconazole versus a regimen of amphotericin B followed by fluconazole for candidaemia in nonneutropenic patients: a randomised non-inferiority trial. Lancet $366,1435-1442$

49 Mora-Duarte, J. et al. (2002) Comparison of caspofungin and amphotericin B for invasive candidiasis. N. Engl. J. Med. 347, 2020-2029

50 Garcia-Effron, G. et al. (2008) A naturally occurring proline-to-alanine amino acid change in Fks1p in Candida parapsilosis, Candida orthopsilosis, and Candida metapsilosis accounts for reduced echinocandin susceptibility. Antimicrob. Agents Chemother. 52, 2305-2312

51 Pappas, P.G. et al. (2007) Micafungin versus caspofungin for treatment of candidemia and other forms of invasive candidiasis. Clin. Infect. Dis. 45, 883-893

52 Park, S. et al. (2005) Specific substitutions in the echinocandin target Fks1p account for reduced susceptibility of rare laboratory and clinical Candida sp. isolates. Antimicrob. Agents Chemother. 49, 3264-3273

53 Balashov, S.V. et al. (2006) Assessing resistance to the echinocandin antifungal drug caspofungin in Candida albicans by profiling mutations in FKS1. Antimicrob. Agents Chemother. 50, 2058-2063

54 Garey, K.W. et al. (2006) Time to initiation of fluconazole therapy impacts mortality in patients with candidemia: a multi-institutional study. Clin. Infect. Dis. 43, 25-31

55 Baquir, B. et al. (2010) Immunological reactivity of blood from healthy humans to the rAls3p-N vaccine protein. J. Infect. Dis. 201, 473-477 
56 Kauffman, C.A. et al. (2000) Prospective multicenter surveillance study of funguria in hospitalized patients. The National Institute for Allergy and Infectious Diseases (NIAID) Mycoses Study Group. Clin. Infect. Dis. 30, 14-18

57 Kobayashi, C.C. et al. (2004) Candiduria in hospital patients: a study prospective. Mycopathologia 158, 49-52

58 Hope, W.W. (2009) Assessing candiduria in a critically ill patient. $\mathrm{Bmj}$ $338, \mathrm{~b} 2289$

59 Tuon, F.F. et al. (2009) Bladder irrigation with amphotericin B and fungal urinary tract infection-systematic review with meta-analysis. Int. J. Infect. Dis. 13, 701-706

60 Sobel, J.D. et al. (2000) Candiduria: a randomized, double-blind study of treatment with fluconazole and placebo. The National Institute of Allergy and Infectious Diseases (NIAID) Mycoses Study Group. Clin. Infect. Dis. 30, 19-24

61 Lundstrom, T. and Sobel, J. (2001) Nosocomial candiduria: a review. Clin. Infect. Dis. 32, 1602-1607

62 Hamza, O.J. et al. (2008) Single-dose fluconazole versus standard 2week therapy for oropharyngeal candidiasis in HIV-infected patients: a randomized, double-blind, double-dummy trial. Clin. Infect. Dis. 47, $1270-1276$

63 Villanueva, A. et al. (2001) A randomized double-blind study of caspofungin versus amphotericin for the treatment of candidal esophagitis. Clin. Infect. Dis. 33, 1529-1535

64 de Wet, N. et al. (2004) A randomized, double-blind, parallel-group, dose-response study of micafungin compared with fluconazole for the treatment of esophageal candidiasis in HIV-positive patients. Clin. Infect. Dis. 39, 842-849

65 Krause, D.S. et al. (2004) A randomized, double-blind trial of anidulafungin versus fluconazole for the treatment of esophageal candidiasis. Clin. Infect. Dis. 39, 770-775

66 Sawyer, S.M. et al. (1994) Vulvovaginal candidiasis in young women with cystic fibrosis. Bmj 308, 1609

67 Sobel, J.D. et al. (2003) Treatment of vaginitis caused by Candida glabrata: use of topical boric acid and flucytosine. Am. J. Obstet. Gynecol. 189, 1297-1300

68 White, D.J. et al. (2001) Combined topical flucytosine and amphotericin B for refractory vaginal Candida glabrata infections. Sex Transm. Infect. 77, 212-213

69 Moudgal, V.V. and Sobel, J.D. (2003) Antifungal drugs in pregnancy: a review. Expert Opin. Drug Saf. 2, 475-483

70 Verweij, P.E. et al. (2009) Azole resistance in Aspergillus fumigatus: a side-effect of environmental fungicide use? Lancet Infect. Dis. 9, 789-795

71 Howard, S.J. et al. (2009) Frequency and evolution of Azole resistance in Aspergillus fumigatus associated with treatment failure. Emerg. Infect. Dis. 15, 1068-1076

72 Denning, D.W. and Stevens, D.A. (1990) Antifungal and surgical treatment of invasive aspergillosis: review of 2,121 published cases. Rev. Infect. Dis. 12, 1147-1201

73 Bates, D.W. et al. (2001) Mortality and costs of acute renal failure associated with amphotericin B therapy. Clin. Infect. Dis. 32, 686-693

74 Wingard, J.R. et al. (1999) Clinical significance of nephrotoxicity in patients treated with amphotericin B for suspected or proven aspergillosis. Clin. Infect. Dis. 29, 1402-1407

75 Ellis, M. et al. (1998) An EORTC international multicenter randomized trial (EORTC number 19923) comparing two dosages of liposomal amphotericin B for treatment of invasive aspergillosis. Clin. Infect. Dis. 27, 1406-1412

76 Denning, D.W. et al. (1994) NIAID Mycoses Study Group multicenter trial of oral itraconazole therapy for invasive aspergillosis. Am. J. Med. 97, 135-144

77 Herbrecht, R. et al. (2002) Voriconazole versus amphotericin B for primary therapy of invasive aspergillosis. N. Engl. J. Med. 347, 408-415

78 Nivoix, Y. et al. (2008) Factors associated with overall and attributable mortality in invasive aspergillosis. Clin. Infect. Dis. 47, $1176-1184$
79 Upton, A. et al. (2007) Invasive aspergillosis following hematopoietic cell transplantation: outcomes and prognostic factors associated with mortality. Clin. Infect. Dis. 44, 531-540

80 Wheeler, R.T. and Fink, G.R. (2006) A drug-sensitive genetic network masks fungi from the immune system. PLoS Pathog 2, e35

$81 \mathrm{Hohl}$, T.M. et al. (2008) Caspofungin modulates inflammatory responses to Aspergillus fumigatus through stage-specific effects on fungal beta-glucan exposure. J. Infect. Dis. 198, 176-185

82 Lamaris, G.A. et al. (2008) Caspofungin-mediated beta-glucan unmasking and enhancement of human polymorphonuclear neutrophil activity against Aspergillus and non-Aspergillus hyphae. J. Infect. Dis. 198, 186-192

83 Ouchi, H. et al. (2007) Successful treatment of refractory chronic necrotizing pulmonary aspergillosis with micafungin. J. Infect. Chemother. 13, 258-262

84 Denning, D.W. et al. (2003) Chronic cavitary and fibrosing pulmonary and pleural aspergillosis: case series, proposed nomenclature change, and review. Clin. Infect. Dis. 37 (Suppl 3), S265-S280

85 Camuset, J.et al. (2007) Treatment of chronic pulmonary aspergillosis by voriconazole in nonimmunocompromised patients. Chest 131, 1435-1441

86 Crosdale, D.J. et al. (2001) Mannose-binding lectin gene polymorphisms as a susceptibility factor for chronic necrotizing pulmonary aspergillosis. J. Infect. Dis. 184, 653-656

87 Sambatakou, H. et al. (2006) Cytokine profiling of pulmonary aspergillosis. Int. J. Immunogenet. 33, 297-302

88 Vaid, M. et al. (2007) Distinct alleles of mannose-binding lectin (MBL) and surfactant proteins A (SP-A) in patients with chronic cavitary pulmonary aspergillosis and allergic bronchopulmonary aspergillosis. Clin. Chem. Lab. Med. 45, 183-186

89 Jain, L.R. and Denning, D.W. (2006) The efficacy and tolerability of voriconazole in the treatment of chronic cavitary pulmonary aspergillosis. J. Infect. 52, e133-137

90 Snelders, E. et al. (2008) Emergence of azole resistance in Aspergillus fumigatus and spread of a single resistance mechanism. PLoS Med. 5, e219

91 Stevens, D.A. et al. (2000) A randomized trial of itraconazole in allergic bronchopulmonary aspergillosis. N. Engl. J. Med. 342, 756762

92 Wark, P.A. et al. (2003) Anti-inflammatory effect of itraconazole in stable allergic bronchopulmonary aspergillosis: a randomized controlled trial. J. Allergy Clin. Immunol. 111, 952-957

93 Denning, D.W. et al. (2009) Randomized controlled trial of oral antifungal treatment for severe asthma with fungal sensitization: The Fungal Asthma Sensitization Trial (FAST) study. Am. J. Respir. Crit. Care Med. 179, 11-18

94 Longley, N. et al. (2008) Dose response effect of high-dose fluconazole for HIV-associated cryptococcal meningitis in southwestern Uganda. Clin. Infect. Dis. 47, 1556-1561

95 Nussbaum, J.C. et al. (2010) Combination flucytosine and high-dose fluconazole compared with fluconazole monotherapy for the treatment of cryptococcal meningitis: a randomized trial in Malawi. Clin. Infect. Dis. 50, 338-344

96 Roden, M.M. et al. (2005) Epidemiology and outcome of zygomycosis: a review of 929 reported cases. Clin. Infect. Dis. 41, 634-653

97 Kontoyiannis, D.P. et al. (2005) Zygomycosis in a tertiary-care cancer center in the era of Aspergillus-active antifungal therapy: a casecontrol observational study of 27 recent cases. J. Infect. Dis. 191, $1350-1360$

98 Reed, C. et al. (2008) Combination polyene-caspofungin treatment of rhino-orbital-cerebral mucormycosis. Clin. Infect. Dis. 47, 364-371

99 Spellberg, B. et al. (2009) Safety and outcomes of open-label deferasirox iron chelation therapy for mucormycosis. Antimicrob. Agents Chemother. 53, 3122-3125

100 Ben-Ami, R. et al. (2009) Phaeohyphomycosis in a tertiary care cancer center. Clin. Infect. Dis. 48, 1033-1041 